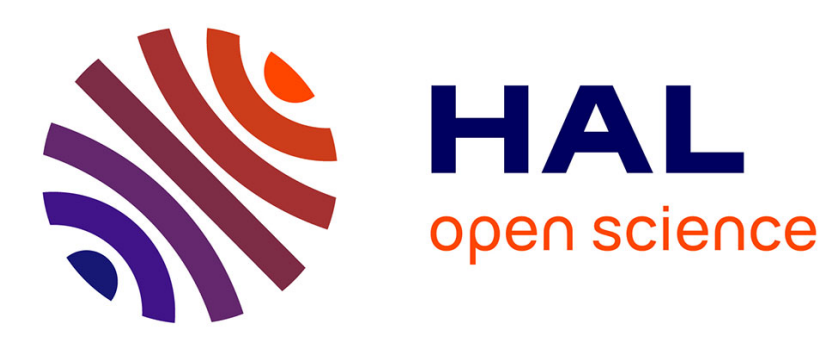

\title{
The role of medical imaging in the context of photodynamic therapy
}

C. Dupont, M. Vermandel, Serge Mordon

\section{To cite this version:}

C. Dupont, M. Vermandel, Serge Mordon. The role of medical imaging in the context of photodynamic therapy. 17th International Photodynamic Association World Congress, 2019, Jun 2019, Cambridge, Massachusetts, United States. 10.1117/12.2528007 . hal-02879034

\section{HAL Id: hal-02879034 \\ https://hal.science/hal-02879034}

Submitted on 23 Jun 2020

HAL is a multi-disciplinary open access archive for the deposit and dissemination of scientific research documents, whether they are published or not. The documents may come from teaching and research institutions in France or abroad, or from public or private research centers.
L'archive ouverte pluridisciplinaire HAL, est destinée au dépôt et à la diffusion de documents scientifiques de niveau recherche, publiés ou non, émanant des établissements d'enseignement et de recherche français ou étrangers, des laboratoires publics ou privés. 


\title{
The role of medical imaging in the context of photodynamic therapy
}

\author{
C. Dupont*a ${ }^{* a}$ M. Vermandel ${ }^{\mathrm{a}}$, S. Mordon ${ }^{\mathrm{a}}$ \\ aUniv. Lille, Inserm, CHU Lille, U1189 - ONCO-THAI - Image Assisted Laser Therapy for Oncology, F- \\ 59000 Lille, France
}

\begin{abstract}
Photodynamic therapy (PDT) is a modality with promising results for the treatment of various cancers. PDT is increasingly included in the standard of care for different pathologies. The role of medical imaging in this context is crucial to better understand how and where to deliver the therapy but also to observe the different mechanisms involved in the effects on tumors.

At different stages of delivery, PDT requires imaging to plan, evaluate and monitor treatment. In this paper, we review the contribution of Magnetic Resonance Imaging or Positron Emission Tomography for planning and therapeutic monitoring purposes.

Several solutions have been proposed to plan PDT from imaging. MRI and dedicated segmentation algorithm have been recently proposed to plan interstitial PDT with stereotactic localization and light diffusion simulation capabilities. Additionally, photosensitizer biodistribution has been evaluated with radiolabeled photosensitizers. The effects of PDT delivery have also been explored with specific Magnetic Resonance Imaging or Positron Emission Tomography radiopharmaceuticals to evaluate the effects on cells (apoptosis, necrosis, proliferation, metabolism) or vascular damage.

The contribution of medical imaging in the context of photodynamic therapies is important and continues to increase. Using morphological or molecular imaging has to be considered for future developments of PDT.
\end{abstract}

\section{Keywords}

photodynamic therapy, medical imaging, treatment planning system, monitoring 


\section{INTRODUCTION}

Although currently recommended as the first-line treatment for actinic keratosis, Bowen's disease and superficial basal cell carcinoma [1], Photodynamic therapy (PDT) remains an experimental option in other oncological indications [2]. During the last decades, several attempts have successfully demonstrated a significant impact on the treatment of various cancers. The practice of PDT in dermatological indications compared to other therapeutic targets could be explained by its straightforward implementation in patient management. Monitoring the PDT effect on lesions can be visually assessed using visible light or fluorescence diagnosis system [3].

Due to the growing interest of PDT in oncology, other cancers are focused. Contrary to dermatology, these other applications are unreachable topically. Consequently, light sources have to be placed near or inside the targeted lesions to produce the expected photoreaction between photosensitizer (PS) and oxygen that will destroy cells with a high PS uptake. Monitoring such a therapy cannot be performed visually and requires the use of non-invasive imaging technics.

In this context, the role of medical imaging appears necessary and should be integrated in all steps of PDT treatments: planning, intraoperative, monitoring and follow-up. Besides the clinical purpose to monitor the disease progression, medical imaging could expand the understanding of biological processes involved in PDT effects on tumors and improve its delivery. Results of preclinical studies should provide clinical tools to define radiological markers of PDT efficacy in order to support the optimization of the treatment delivery and the design of future PDT clinical trials. In this proceeding, we will introduce the role of medical imaging during all steps of the patient care, through our experience in the development of PDT for the treatment of brain cancer.

\section{PLANNING}

For mini-invasiveness purpose, optical fibers are inserted interstitially for delivering laser light inside the tumor tissues. A strong effort has been achieved to develop treatment planning systems (TPS) dedicated to different treatment of cancers (prostate [4-6], mesothelioma [7, 8], glioblastoma $[9$, 10]). In most of the cases, $C T$ and MRI imaging are used to plan the insertion point, direction and final location of optical fibers. CT imaging provides structural data from optical density of encountered organs used to compute cavity volumes or optimize location of light sources insertion. Multiparametric MRI offers multiple sequences to study the functional activities of structures and to obtain a better contrast between soft tissues. 
In a PDT dosimetric context, several light transport models are used to compute the laser light propagation in tissues surrounding light sources and to compute a light dose. Analytic equations, finite element method or Monte-Carlo algorithm can be employed to perform this dosimetry. However, all these models require optical properties of tissues, such as absorption and scattering coefficient, anisotropic and refractive index in their computation input.

Although MRI imaging does not offer a direct value of these optical properties, using segmentation or classification algorithms [11], each voxel constituting the MRI volume can be associated with optical properties. Thus, preoperative imaging can be employed to take into account the strong heterogeneity of tissues and deliver a PDT planning of interstitial procedure with a patient-specific dosimetry [12].

Recently, molecular imaging such as Single photon emission computed tomography (SPECT) and positron emission tomography (PET) provides a promising outcome in the PDT management. The effects of PDT delivery on cells (apoptosis, necrosis, proliferation, metabolization) or vascular damages have been explored using these technologies [13]. PS biodistribution obtained from these imaging could integrate future dosimetric computation in order to take into account insufficient or heterogeneous PS accumulation in tumor tissues. Moreover, imaging hypoxia prior to the PDT delivery could be a relevant feature to integrate into a dosimetric computation.

\section{INTRAOPERATIVE}

PDT is a therapy based on three components: light, PS and oxygen. It appears necessary to assess these three components to tend towards a complete comprehension of PDT effect. A strong effort has been performed to monitor PDT using variation of oxygen level with photoacoustic, PS fluorescence and singlet oxygen phosphorescence signals during treatments [14-18]. These measurements are implemented with probes inserted in tissues and recent theranostics technologies have been developed to combine both illumination source and spectroscopy detection system $[19,20]$.

Additionally, fluorescence properties of PS are exploited during resection surgery as "fluorescenceguided resection" technic [21]. By lighting the operating field with a wavelength matching with a PS's absorption peak, a fluorescence signal is reemitted. With an appropriate PS allowing a strong selectivity of tumor cells, areas showing a visual fluorescence lightning guides the neurosurgeon during the procedure and help to discriminate healthy from reaming tumor tissue. This surgical technique demonstrates an improvement of the extent of resection, which is a major prognostic factor in patients' survival. 5-ALA has recently been approved for high-grade gliomas surgery as an intraoperative optical imaging fluorescent agent in the US [22]. 
Although few centers possess such a device, Intraoperative MRI can provide relevant data regarding the resection quality. This imaging technic allows to control whether the surgery can be considered as radiologically complete or the resection can be pursuing on tumor remnants. Most of the time, gross total resection and extent of resection are enhanced and patients' survival (progression free survival and overall survival) are increased [21].

To date, intraoperative medical imaging does not appear as a major contributor in the PDT field. However, the clinical trial INDYGO has recently included intraoperative MRI in the workflow in order to detect prospective early pattern of PDT effect [23]. 10 patients have been treated for their brain tumor using intraoperative PDT and the follow-up is still active.

\section{FOLLOW-UP}

MRI imaging stands for the prime candidate to follow patients' diseases. Primary effects of PDT are necrosis and apoptosis of tumor cells and the reduction of tumor vascularization. Our previous preclinical studies allowed us to compare MRI volumes with histological samples [24-26]. Using these results, we demonstrated that diffusion MRI is a marker of cell death, perfusion MRI indicates the level of necrosis and $T 2$ sequences enhance strongly the peritumoral edema of tumors.

Based on these results, we performed a meta-analysis to investigate the link between molecular (histology) and functional imaging (MRI) [27]. We evaluated the impact of different PDT treatment schemes on Forty-eight "nude" rats grated with brain cancers cells. Four different treated groups have been shaped: low laser power, high laser power, two fractions and five fractions of the entire lighting duration required to obtain a given light dose $\left(25 \mathrm{~J} / \mathrm{cm}^{2}\right)$.

Using the complementary of MRI signals and histological samples, several conclusions have been drawn. High laser power induced a strong intratumoral necrosis with a strong surrounding edema and inflammatory response. Low laser power tends to induce more apoptosis than necrosis, which causes less damages to healthy adjacent tissues than necrosis. Concerning the fractionation, the five-fractions scheme was more effective than the two-fraction scheme regarding the level of induced necrosis but also with a stronger peritumoral edema. These results were in accordance with studies on oxygen level showing that the efficacy of PDT treatments are highly related to the initial oxygen concentration [28].

In view of these elements, studies on MRI radiomics post-PDT treatment should be determinant to assess the quality of the PDT treatment delivered and could bring quantitative criteria during patient's follow-up. 


\section{DISCUSSION}

PDT should benefit from medical imaging to enhance both the understanding of biological processes involved during treatment and define criteria to evaluate the quality of tumor's response.

Medical imaging should integrate fully clinical trials and support their design. As mentioned before, the INDYGO trial integrated both intraoperative MRI study, the research of radiomics during the follow-up phase and the five-fraction illumination scheme arising from our preclinical MRI and histological studies.

Additionally, PET imaging may provide an added value in terms of PS biodistribution and tumor activity after a PDT treatment. Radiolabeled photosensitizers are currently being developed to integrate molecular imaging to the medical imaging technologies available in clinical practice.

\section{REFERENCES}

[1] C. A. Morton, R.-M. Szeimies, A. Sidoroff et al., "European guidelines for topical photodynamic therapy part 1: treatment delivery and current indications - actinic keratoses, Bowen's disease, basal cell carcinoma," Journal of the European Academy of Dermatology and Venereology, 27(5), 536-544 (2013).

[2] C. Frochot, and S. Mordon, "Update of the situation of clinical photodynamic therapy in Europe in the 2003-2018 period," Journal of Porphyrins and Phthalocyanines, 23(04n05), 347-357 (2019).

[3] J. Tyrrell, S. Campbell, and A. Curnow, "Validation of a non-invasive fluorescence imaging system to monitor dermatological PDT," Photodiagnosis and Photodynamic Therapy, 7(2), 86-97 (2010).

[4] J. Cassidy, V. Betz, and L. Lilge, "Treatment plan evaluation for interstitial photodynamic therapy in a mouse model by Monte Carlo simulation with FullMonte," Frontiers in Physics, 3, 1-10 (2015).

[5] S. R. Davidson, R. A. Weersink, M. A. Haider et al., "Treatment planning and dose analysis for interstitial photodynamic therapy of prostate cancer," Phys Med Biol, 54(8), 2293-313 (2009).

[6] A. Rendon, J. C. Beck, and L. Lilge, "Treatment planning using tailored and standard cylindrical light diffusers for photodynamic therapy of the prostate," Phys Med Biol, 53(4), 1131-49 (2008). 
[7] W. Brahim, M. Mestiri, N. Betrouni et al., "Malignant pleural mesothelioma segmentation for photodynamic therapy planning," Comput Med Imaging Graph, 65, 79-92 (2018).

[8] J. Sandell, C. Chang, J. C. Finlay et al., "A Treatment Planning System for Pleural PDT," Proceedings of SPIE--the International Society for Optical Engineering, 7551, 75510C (2010).

[9] A. A. Yassine, W. Kingsford, Y. Xu et al., "Automatic interstitial photodynamic therapy planning via convex optimization," Biomed Opt Express, 9(2), 898-920 (2018).

[10] C. Dupont, N. Betrouni, S. R. Mordon et al., "5-ALA Photodynamic Therapy in Neurosurgery, Towards the Design of a Treatment Planning System: A Proof of Concept," IRBM, 38(1), 34-41 (2017).

[11] C. Dupont, N. Betrouni, N. Reyns et al., "On Image Segmentation Methods Applied to Glioblastoma: State of Art and New Trends," IRBM, 37(3), 131-143 (2016).

[12] B. W. Pogue, J. T. Elliott, S. C. Kanick et al., "Revisiting photodynamic therapy dosimetry: reductionist \& surrogate approaches to facilitate clinical success," Phys Med Biol, 61(7), R5789 (2016).

[13] D. Kharroubi Lakouas, D. Huglo, S. Mordon et al., "Nuclear medicine for photodynamic therapy in cancer: planning, monitoring and nuclear PDT," Photodiagnosis and Photodynamic Therapy, 18, 17 (2017).

[14] B. Q. S. Jonathan P. Celli, Imran Rizvi,Conor L. Evans, Kimberley S. Samkoe, Sarika Verma,, and a. T. H. Brian W. Pogue, "Imaging and Photodynamic Therapy: Mechanisms, Monitoring, and Optimization," Chemical Reviews, 110(5), 44 (2010).

[15] J. P. Celli, B. Q. Spring, I. Rizvi et al., "Imaging and Photodynamic Therapy: Mechanisms, Monitoring, and Optimization," Chemical Reviews, 110(5), 2795-2838 (2010).

[16] M. M. Kim, R. Penjweini, N. R. Gemmell et al., "A Comparison of Singlet Oxygen Explicit Dosimetry (SOED) and Singlet Oxygen Luminescence Dosimetry (SOLD) for PhotofrinMediated Photodynamic Therapy," Cancers, 8(12), 109 (2016).

[17] P. Shao, D. W. Chapman, R. B. Moore et al., "Monitoring photodynamic therapy with photoacoustic microscopy," J Biomed Opt, 20(10), 106012 (2015).

[18] J. Jo, C. H. Lee, R. Kopelman et al., "Lifetime-resolved Photoacoustic (LPA) Spectroscopy for monitoring Oxygen change and Photodynamic Therapy (PDT)," Proceedings of SPIE--the International Society for Optical Engineering, 9708, 97081L (2016). 
[19] J. Desroches, M. Jermyn, M. Pinto et al., "A new method using Raman spectroscopy for in vivo targeted brain cancer tissue biopsy," Sci Rep, 8(1), 1792 (2018).

[20] S. Y. Li, H. Cheng, B. R. Xie et al., "A ratiometric theranostic probe for tumor targeting therapy and self-therapeutic monitoring," Biomaterials, 104, 297-309 (2016).

[21] E. Suero Molina, S. Schipmann, and W. Stummer, "Maximizing safe resections: the roles of 5aminolevulinic acid and intraoperative MR imaging in glioma surgery-review of the literature," Neurosurg Rev, (2017).

[22] C. G. Hadjipanayis, and W. Stummer, "5-ALA and FDA approval for glioma surgery," Journal of Neuro-Oncology, 141(3), 479-486 (2019).

[23] C. Dupont, M. Vermandel, H. A. Leroy et al., "INtraoperative photoDYnamic Therapy for GliOblastomas: Study Protocol for a Phase I Clinical Trial," Neurosurgery, nyy324-nyy324 (2018).

[24] H. A. Leroy, M. Vermandel, B. Leroux et al., "MRI assessment of treatment delivery for interstitial photodynamic therapy of high-grade glioma in a preclinical model," Lasers Surg Med, (2017).

[25] H. A. Leroy, M. Vermandel, A. S. Vignion-Dewalle et al., "Interstitial photodynamic therapy and glioblastoma: Light fractionation in a preclinical model," Lasers Surg Med, 49(5), 506-515 (2017).

[26] M. C. Tetard, M. Vermandel, H. A. Leroy et al., "Interstitial 5-ALA photodynamic therapy and glioblastoma: preclinical model development and preliminary results," Photodiagnosis Photodyn Ther, (2015).

[27] M. Vermandel, M. Quidet, A.-S. Vignion-Dewalle et al., "Comparison of different treatment schemes in 5-ALA interstitial photodynamic therapy for high-grade glioma in a preclinical model: An MRI study," Photodiagnosis and Photodynamic Therapy, (2018).

[28] G. Kareliotis, S. Liossi, and M. Makropoulou, "Assessment of singlet oxygen dosimetry concepts in photodynamic therapy through computational modeling," Photodiagnosis Photodyn Ther, (2017). 